Ciencia y Educación, Vol. 6, No. 1, enero-abril, 2022

ISSN (impreso): 2613-8794・ISSN (en línea): 2613-8808

DOI: https://doi.org/10.22206/cyed.2022.v6i1.pp119-128

\title{
La relevancia de los lenguajes estéticos en los procesos educativos
}

\author{
The relevance of aesthetic languages \\ in educational processes
}

\author{
Camila Fernández de Córdova ${ }^{1}$ ORCID: 0000-0002-1533-0817 \\ Enrique Mediavilla Naranjo² ORCID: 0000-0001-7786-1077
}

Recibido: 14/08/2021 • Aprobado: 20/10/2021

Cómo citar: Fernández De Córdova, C., \& Mediavilla Naranjo, E. La relevancia de los lenguajes estéticos en los procesos educativos. Ciencia y Educación, 6(1), 119-128. https://doi.org/10.22206/cyed.2022.v6i1.pp119-128

\begin{abstract}
Resumen
La puesta en valor del universo de los lenguajes estéticos con relación a las necesidades de la sociedad contemporánea y el permanente cuestionamiento de la vigencia de las estructuras educativas tradicionales desemboca en la búsqueda de nuevas alternativas pedagógicas. En este sentido, se reflexiona sobre la necesidad de entretejer los ámbitos artísticos, simbólicos y estéticos con las diversas áreas de aprendizaje, a la luz de perspectivas que tengan la intención de resignificar las dimensiones que configuran los procesos educativos. Además, se vislumbra la importancia de generar oportunidades formativas que atiendan a la figura docente con la intención de que se establezca como el vector fundamental de transformación y mejora de la calidad educativa, a partir de las experiencias cognitivas y emocionales que se generan desde la expresión estética.
\end{abstract}

Palabras clave: artes, educación artística, educación estética, formación de docentes, lenguaje simbólico.

\begin{abstract}
The enhancement of the universe of aesthetic languages in relation to the needs of contemporary society and the permanent questioning of the validity of traditional educational structures, leads to the search for new pedagogical alternatives. In this sense, it reflects on the need to interweave the artistic, symbolic, and aesthetic fields with the various areas of learning, in the light of perspectives that intend to resignify the dimensions that build up the educational processes. In addition, it is foreseen the importance of generating training opportunities that addresses the teaching figure with the intention of establishing itself as the fundamental vector of transformation and improvement of educational quality, based on the cognitive and emotional experiences that are generated from the aesthetic experience.
\end{abstract}

Keywords: Arts, art education, aesthetic education, teacher education, symbolic languages.

\footnotetext{
a Investigadora independiente. Correo-e: camilunas@hotmail.com

b Universidad de Granada, España. Correo-e: enriquemediavil@gmail.com
} 


\section{Introducción}

Si bien en la actualidad se ha evidenciado una reivindicación del acto creador como indispensable para afrontar los múltiples retos y desafíos que surgen permanentemente en los diversos contextos del quehacer humano, de todas maneras, las artes, consideradas esenciales para el desarrollo de las capacidades creativas, continúan teniendo una posición periférica frente a otras disciplinas que se consideran de mayor relevancia.

Cabe señalar que, si bien, la interpretación y la expresión a través de los diferentes lenguajes estéticos no es exclusiva de las artes como disciplinas desconectadas y de la colectividad de artistas, sino que compete de manera abarcadora a las distintas esferas de la humanidad. Esto se debe a que son, fundamentalmente, "un proceso de empatía que relaciona al yo con las cosas y a las cosas entre sí" (Vecchi, 2013, p. 58), lo que les confiere la capacidad de atravesar todos los ámbitos pedagógicos como activadores del aprendizaje.

De todas maneras, hace falta promover vivencias y experimentaciones que partan de la educación artística porque en ellas subyace la esencia liberadora de estos lenguajes, tal y como lo menciona Rodari (2001), en su famosa frase publicada en 1973 en la Gramática de la fantasía, en referencia a la democratización de la palabra: "No para que todos sean artistas, sino para que nadie sea esclavo" (p. 12).

En esta dirección, se demandan miradas y acciones guiadas por un pensamiento renovador y complejo, que sea capaz de ir más allá de un desempeño restringido a las estructuras clásicas que han marcado la generación del conocimiento, para plantear relaciones dialógicas entre los distintos saberes que dan lugar a propuestas transformadoras (Morin, 1999).

Desde estas premisas, se requiere de una comunidad con un verdadero compromiso social, dispuesta a afrontar cambios y a sembrar conciencia sobre la necesidad de dar respuestas colectivas a los permanentes cuestionamientos que emergen de la realidad. "En este sentido, las propuestas educativas han de procurar que la vida entre y salga del aula de la mano del educador, de los propios alumnos y del conjunto de la comu- nidad" (Martínez Vérez et al., 2017, p. 110). En virtud de esto, se deben plantear acciones pedagógicas que estén preocupadas por un desarrollo humano e integral, capaces de converger en la generación de capacidades éticas y reflexivas destinadas al bien común.

De esta forma, se encuentra pertinente recurrir a espacios que estén caracterizados por matices y propuestas que se orienten a la pedagogía de lo sensible, es decir, que se ocupen de otras dimensiones del ser humano y se salgan de los parámetros considerados dentro de la normalidad (Planella, 2017). En ese sentido, se plantea una reflexión sobre la relevancia de atender a aquellas manifestaciones provenientes de los diversos lenguajes estéticos y a sus potencialidades, que se relacionan y asocian con el universo educativo. No se trata de enfatizar las posibilidades de las artes como una asignatura aislada, sino de entender que ciertas características inherentes a la educación artística enriquecen los procesos pedagógicos de una manera holística, que transciende a los límites de las diversas disciplinas.

Por consiguiente, si se considera a la figura docente como una de las claves para la mejora de la calidad de las propuestas educativas, se deben propiciar procesos experimentales y teóricos que logren sensibilizar al colectivo de educadores sobre los beneficios que se presentan en la relación existente entre el mundo estético y el pedagógico.

\section{La necesidad de reflexionar sobre los lenguajes estéticos}

El desarrollo comunitario tiene como una de sus bases fundamentales la naturaleza relacional del ser humano. Si esta premisa de partida se ubica en una perspectiva pedagógica, se tiene que atender con especial cuidado al fomento de las capacidades comunicativas, colaborativas y expresivas. Además, desde la configuración de cada parcela de la sociedad se debe apostar por brindar espacios y oportunidades en los que coexistan distintas narrativas particulares para que se puedan entretejer de una manera armónica y natural en diálogos en pro de la cultura de paz (Sánchez-Fernández et al., 2019). 
Desde este enfoque, la prioridad estriba en impulsar visiones genuinas sobre la percepción de la realidad o del mundo y, al mismo tiempo, ponerlas en interconexión, de forma constructiva y reflexiva, con el resto de subjetividades, a través de múltiples maneras de comunicar. En ese contexto, los lenguajes son entidades que están dotadas de cualidades que van más allá de la mera transmisión específica de signos o mensajes, para pasar a configurarse como sistemas complejos y dinámicos, relevantes en la cohesión y transformación de las sociedades.

Por lo tanto, el lenguaje se constituye como un elemento de acción (Ros, 2004) y se reconoce la existencia de un amplio abanico de posibilidades, que están determinadas por el origen y las intencionalidades de cada mensaje, la forma en la que se produce y los distintos niveles de interpretación. Consecuentemente, se encuentra pertinente propiciar espacios de reflexión que atiendan a los diversos lenguajes en un doble sentido: por un lado, desde la forma, si se enfoca la atención en las múltiples maneras en las que se manifiestan; y, por otro, desde el contenido, en la particularidad que nace de forma intrínseca en ellos, a partir de la capacidad de cada ser humano.

Por consiguiente, se evidencia la necesidad de que las reflexiones sobre la relevancia de las diversas potencialidades expresivas de los lenguajes tengan resonancia en el quehacer educativo. En esta línea de razonamiento, Mediavilla (2021) realza las oportunidades pedagógicas de las artes para favorecer procesos integrales de desarrollo.

Los lenguajes artísticos se caracterizan por su ductilidad, creatividad, complejidad, por la capacidad de encontrar diálogos desde una multiplicidad de miradas y sentidos, por su ruptura de la cotidianidad, generar grandes espacios interpretativos y su pretensión de transcender en el tiempo. (p. 27)

Además, la educación artística contemporánea plantea diversas manifestaciones de los lenguajes estéticos orquestados en las artes plásticas, escénicas, musicales, literarias, audiovisuales, entre otras, que se presentan a través de propuestas alternativas y/o perfor- máticas, propias de la época posmoderna (Efland et al., 2003). Estas expresiones culturales suponen una oportunidad, ya que se difuminan las líneas divisorias que separaban al grupo de espectadores del de creadores al poner en valor la experiencia comunitaria, las maneras de comunicación y, por tanto, las relaciones dialógicas entre los colectivos, todos ellos factores enriquecedores y relevantes para los procesos pedagógicos.

En ese contexto, se debe señalar que, con relación a los lenguajes artísticos, el concepto de estética puede tener diversas aproximaciones. Eco (2007), por ejemplo, establece que los idearios occidentales de lo bello o lo feo cambian de sentido según la historia o las diversas culturas y que, en algunos casos, atienden a criterios políticos y sociales. Por lo tanto, se requiere precisar que es pertinente adoptar una perspectiva amplia que no se circunscriba únicamente a la dualidad entre belleza y fealdad, sino que admita "una filosofía de lo sensible" (Caro, 2014, p. 9).

En este orden de ideas, Farina (2006) escribe sobre una dimensión estética conformada por componentes éticos y políticos, que se desarrolla a través de acontecimientos que, de alguna forma, desestabilizan el mundo cotidiano. Sin embargo, gracias a esta afectación de la cotidianidad, se pueden producir nuevos planteamientos y una conciencia renovada. En otros términos, el mundo estético hace posible que se generen cuestionamientos que emergen de distintas y nuevas percepciones, relacionadas con el lugar o el papel que cada uno ocupa o desempeña y por las influencias que gravitan en el entorno.

Al hilo de la reflexión que propone Farina, sobre los modos de acercar las prácticas pedagógicas a las prácticas estéticas mediante la propuesta que denomina como pedagogía de las afecciones, se vislumbra el potencial educativo que existe en la gramática particular de los diversos lenguajes, es decir, en sus estructuras comunicacionales complejas y sus infinitas interconexiones, que inciden en la manera en la que se analizan las problemáticas, se configura el pensamiento y la construcción de la percepción del universo.

En consecuencia, se precisa reflexionar con profundidad sobre las maneras de dar cabida a espacios que permitan la manifestación y el desarrollo de las 
potencialidades de los diversos lenguajes estéticos, en beneficio de procesos y proyectos educativos enriquecedores, que estén en consonancia con las necesidades y los requerimientos de la sociedad actual.

\section{La resignificación de los contenidos curriculares a la luz de la educación artística}

Todo proceso educativo se genera a partir de un ejercicio de planificación y transposición didáctica de los saberes. En ese sentido, el currículum oficial funciona como una estructura reguladora que marca el camino y los contenidos que se deben abordar de manera ordenada y legitimada. De alguna manera, el hecho de tener una ruta trazada puede incidir en que los procesos se vuelvan estáticos, fragmentados, unidimensionales, estandarizados y deslindados de su contexto (Sánchez-Carreño \& Pérez-Rodríguez, 2011). No obstante, si se contempla la estructura curricular a la luz del enfoque procesual y transdisciplinar de la educación artística, se posibilitan múltiples dinámicas provocadas por los cuestionamientos, los diálogos, las indagaciones, los hallazgos, las limitaciones y toda una suerte de intersticios que admiten la apertura a distintas temáticas y ramificaciones del conocimiento, de manera compleja. En palabras de Abad (2021), al reflexionar sobre el trabajo de Monclus: "el reto de afrontar la superación de un currículum estable permite adoptar una posición con-nuestro-tiempo que implica no solo un compromiso, sino también un testimonio" (p. 225).

Es decir, se trata de tomar como punto de inicio a las bases del tema previamente establecido, pero con la suficiente apertura para dar cabida al pensamiento estético, que tiene la capacidad de transformar a la práctica pedagógica, paralelamente a las necesidades e inquietudes que surgen de manera espontánea desde la colectividad; idea que queda reflejada con claridad en las palabras de Gómez et al. (2018) al reflexionar sobre la educación poética:

En una educación como fabricación donde predominan ideas dominantes, generalmente, las actividades de aprendizaje ya están previamente establecidas porque se parte de la premisa del control, un hacer poético ha de tener la oportunidad de que el estudiante se ponga a prueba con lo que piensa, lee, escribe, observa, escucha y percibe en la medida en que algo le da a pensar, le genera interrogantes, le produce perturbación y otros gestos que conllevan relaciones de aprendizaje que afiancen la creación, la producción o la poética del sí. (p. 186)

Cabe mencionar que esta suerte de metodología, que parte de preguntas generadoras y de la propia curiosidad y creatividad del grupo de estudiantes, no es potestad de la educación actual, sino que se lleva produciendo en distintos ámbitos de la educación artística y se consolida a inicios del siglo pasado. La pedagogía de la Bauhaus (1919), por ejemplo, que sigue teniendo repercusión en la manera en la que se enseña el diseño a nivel superior, trabaja con una metodología proyectual y está estructurada en clases de tipo taller, en las que el acto creador se percibe desde dimensiones lúdicas y de experimentación, que privilegian al trabajo colaborativo y a las aportaciones interdisciplinarias de los diferentes ámbitos del desarrollo humano: "Se enseńaba a los jóvenes a pensar, a conocerse a sí mismos y a partir de ello a desarrollar su individualidad como creadores originales y auténticos" (Blocona, 2014, p. 89).

Cabe señalar que esta propuesta toma como referencia a algunas corrientes educativas anteriores, entre las que resaltan, en primer lugar, el modelo Fröbeliano, que integra al juego como herramienta pedagógica y al aprendizaje multidisciplinar estructurado en tres esferas, el conocimiento científico, la puesta en valor de lo cotidiano y “... la belleza con la configuración de un lenguaje artístico que engloba el color, la forma y el espacio" (Pozo \& Mayoral, 2017, p. 53); y, en segundo lugar, el método propuesto por Pestalozzi, en el que se potencia una visión analítica y sistémica que da importancia a la forma, en sus dimensiones y proporciones, y al arte del dibujo (Bordes, 2006, p. 10).

En la actualidad, la iniciativa New European Bauhaus (NEB) pretende reflexionar de manera crítica sobre temas sociales, culturales, ecológicos y económicos, 
desde el ámbito educativo, para redefinir y repensar diversos retos relacionados con las problemáticas actuales, buscando conformar un nuevo paradigma en el que el diseño y las artes ocupan un lugar central (Bildi \& Escola Eina, 2020). Esto conlleva a entender que las artes pueden tener una incidencia mayor que la que está delimitada por la propia disciplina.

Así mismo, la propuesta educativa de Reggio Emilia iniciada por Loris Malaguzzi (1920 -1994), recogida en su famoso poema No Way. The Hundred is There, (Sin dudas, los cien están allí), que alude a Los cien lenguajes del niño, y con vigencia en los contextos educativos actuales, resalta la necesidad de ampliar las posibilidades del conocimiento a distintos cauces, generando la reflexión y el cuestionamiento permanente de las ideas. Además, sitúa a las artes en un lugar protagónico con la intención de reconocer y dar cabida a la diversidad de los lenguajes y las formas de expresión, respetando las singularidades de interpretación, representación y relación con el entorno (Martínez-Agut \& Ramos, 2015). De este modo, considera importante que los sistemas educativos sean capaces de ofrecer las herramientas necesarias para tender un puente hacia todas aquellas manifestaciones que generalmente se encuentran relegadas o invisibilizadas detrás de otras áreas u otros objetos del conocimiento (Vecchi, 2013).

Queda reflejado en los ejemplos anteriores que la posibilidad de trabajar con un currículo emergente o vivo, capaz de adaptarse y dar cabida a las diversas inquietudes y maneras de expresión, tal como ocurre en los modelos pedagógicos de la educación artística, puede desembocar en alternativas de amplias posibilidades, no solo a nivel crítico, sino también filosófico y estético y, en definitiva, tener la capacidad de cuestionar y cuestionarse, de construir la identidad en el vaivén de la colectividad, de proponer y disponer para generar conocimiento en aras de los planteamientos y ritmos de la contemporaneidad. Por consiguiente, romper con la estructura rígida de la programación tradicional permite que los ámbitos del saber se transformen y reconfiguren, como lo apunta Caram (2015): "Todo contenido es enriquecido por el grupo que lo estudia, lo reconstruye y resignifica" (p. 93).
Además, en sí mismo, todo acto creador proviene de un marco proyectivo, "un trayecto vivido, no es un instante de un proceso ni un resultado, y como tal, conforma una urdimbre de actos que arrastran una vivencia única y valiosa (...) En sentido estricto no es una experiencia acabada o finita" (Caeiro-Rodríguez, 2018, p. 160), lo que propicia que a partir de las prácticas que provienen de lo que Caeiro-Rodríguez denomina el Aprendizaje Basado en la Creación se configuren y reconfiguren nuevas posibilidades de aprendizaje, ya que no se pueden aplicar los mismos esquemas, modelos, estrategias o metodologías a las distintas personas, situaciones o experiencias adquiridas.

Conviene mencionar que otra de las características de la educación artística, además de potenciar a los diversos lenguajes estéticos, es permitir que se genere una gran interacción o interconexión entre diversas áreas de estudio y traducir el conocimiento a otros formatos retóricos: "Los nuevos tiempos presentan una tendencia clara a no menospreciar los aspectos formales de los discursos (narrados, pintados o filmados), e incluso a considerarlos parte sustancial del contenido" (Diez del Corral, 2005, p. 473). Por tal razón, las artes pueden incidir en la construcción del conocimiento en otras áreas del saber, sin llegar a convertirse en un instrumento utilitario, para dar lugar a una suerte de ósmosis de aprendizajes.

Se trata de un diálogo entre las distintas disciplinas y/o lenguajes que aporta una mayor profundidad a los planos de las diferentes dimensiones estéticas y semióticas, lo que desvanece las fronteras que generalmente estaban establecidas a priori (Marín, 2009). Esta transgresión de los límites entre las distintas áreas del conocimiento genera procesos que dan cabida a manifestaciones pertenecientes a nichos culturales, como lo son la cultura popular, o que se consideran propios de las nuevas tecnologías aplicadas. La ampliación de los niveles de la experiencia estética y de las formas de expresión conlleva a que la interdisciplinariedad de paso a la transdisciplinariedad, lo que consecuentemente redimensiona y resignifica a los procesos formativos: "En la transdisciplinariedad las barreras entre disciplinas desaparecen, ya que cada una reconoce -en su estructura- el carácter de todas las demás” (Hoyuelos, 2013, p. 21). 
Un ejemplo claro en el que la interacción de saberes posibilita la investigación colaborativa es el proyecto Segni Mossi que, en asociación con Mus-e Italia, promueve la inclusión social a través del arte en las escuelas primarias públicas de Roma. Su propuesta busca generar un diálogo equilibrado entre la riqueza semiótica de los lenguajes gráficos y la retórica expresiva de la danza, para dar lugar a distintas vivencias vinculadas a los lenguajes estéticos. El cuerpo se construye en una herramienta comunicativa que explora al signo liberado de lo representativo. En ese contexto, la experimentación se constituye como una manera de indagación que aborda temáticas emergentes como la continuidad, los opuestos, la gravedad, la oscuridad, la inercia, el balance, la simetría, el impulso, la propagación, la deformación, las trayectorias, las redes, la arquitectura, entre otras (Segni Mossi, 2021).

Este tipo de experiencias permite vislumbrar que los horizontes de investigación que se pueden llevar a cabo a través de las artes dependen de la creatividad, el conocimiento de los diversos lenguajes y la necesidad de ampliar los niveles de interpretación de los contenidos que se encuentran legitimados en el currículum, facultades que recaen en la figura del docente, en su libertad de cátedra y en su capacidad de redimensionar la acción educativa.

En ese sentido, se vuelve una necesidad imperante generar acciones formativas que pongan especial énfasis en la formación de formadores, con el objeto de que el cuerpo docente tenga conocimientos experienciales en el quehacer artístico, no desde el punto de vista meramente técnico o utilitario, sino desde la sinergia de los diversos lenguajes estéticos y sus posibilidades de comunicación, reflexión, expresión, interpretación, cuestionamiento, investigación y creación, es decir, todo lo vinculado al desarrollo del pensamiento crítico.

\section{La figura docente como alentadora de la expe- riencia estética}

La necesidad de que el colectivo de educadores conozca criterios artísticos de manera teórica y a través de la experimentación práctica no solo corresponde a la consideración de que las artes posibilitan la interpretación y la configuración de diversos sistemas discursivos y que para enseñarlos hace falta conocerlos a profundidad, sino a que esas experiencias individuales y colectivas permiten ampliar de manera significativa el prisma con el que se percibe la realidad y las aproximaciones hacia los procesos pedagógicos relacionados con las distintas disciplinas, sea cual sea el ámbito del conocimiento.

Por otro lado, atender a la educación artística de manera multidimensional permite tomar conciencia de la brecha que existe entre la pedagogía cultural, que se encarga de la construcción identitaria relacionada con la cultura popular que se encuentra fuera de los lindes de los sistemas educativos, y la pedagogía escolar, determinada por los valores y contenidos que se transmiten dentro de la institucionalidad (González-Méndez, 2017). Como consecuencia, se genera la posibilidad de repercutir en la construcción de procesos pedagógicos que tomen en cuenta todas aquellas experiencias que se producen al margen del centro educativo, no como meros receptores, sino como agentes activos de transformación.

En ese sentido, tal como lo apunta González-Méndez (2017) al reflexionar sobre los postulados de Freedman, las artes aportan al profesorado con propuestas metodológicas, actitudes investigadoras y con elementos teóricos que contribuyen a que el grupo de estudiantes tenga la capacidad de pensar libremente y de manera autónoma:

Así, el papel del docente no ha de ser tanto el de un poseedor de todas las respuestas como el de un garante y facilitador del conocimiento que conduzca al progreso, a la identificación de problemas, al trabajo con las experiencias y a la apertura de posibilidades. (p. 31)

Esta línea de pensamiento y acción supone una invitación dirigida a toda la comunidad educativa a exponerse y generar situaciones educativas de carácter retador e integral, con sentidos estéticos, sociales, culturales y políticos, que estén ligados de manera directa a la trans- 
formación y mejora de su contexto cercano. Desafíos que promueven y precisan poner en relación distintas narrativas y el uso de múltiples lenguajes, con los que hacer significativos y visibles los relatos, las enseñanzas y los aprendizajes colectivos para una comunidad inteligente, ética, justa y solidaria, ideales que encuentran su máxima expresión en el artivismo educativo.

En esta línea de pensamiento, Mesías (2018) razona sobre la manera en que la formación del profesorado en el ámbito artístico incide en los procesos de transformación social; para eso, analiza y comparte diversas propuestas de investigación-acción en donde se conjugan varias facetas del perfil docente como profesor, investigador y artista, en los que se deconstruye el modelo y la figura educadora, para promover la equidad y la justicia social:

Transformar la formación docente desde el artivismo es asumir una ética del riesgo. Es la esperanza radical de creerse capaz de cambiar rutinas, formas de actuación obsoletas, modelos docentes descontextualizados, la resistencia ante las injusticias, desde la innovación, desde las microutopías, para retar y retarnos constantemente... (p. 26)

Por último, es importante considerar que los lenguajes estéticos no solo guardan relación con los aprendizajes académicos o formales, sino que parten de la experiencia o la vivencia de los saberes, lo que significa que se trata de procesos que además de ser cognitivos o metacognitivos también son emocionales o metaemocionales (Caeiro-Rodríguez, 2018). Por lo tanto, garantizar que el colectivo docente, sin importar el área académica a la que pertenezca, se capacite en educación artística implica la posibilidad de que transforme la manera en que se relaciona con el quehacer educativo, logrando que los conceptos concernientes al aprendizaje pasen de estar únicamente vinculados al plano cognitivo a ser criterios de mayor amplitud que permitan la presencia de lo lúdico, lo creativo, lo divergente, lo intuitivo, lo interactivo, lo polivalente, entre otras maneras en las que se da pie al ámbito de lo sensible.

\section{Conclusiones}

Se ha reflexionado sobre las potencialidades de las artes como posibilitadoras de propuestas metodológicas, investigativas, creativas y conceptuales capaces de modificar las prácticas pedagógicas desde un enfoque abarcador, que no se circunscribe específicamente a la disciplina artística per se. Por consiguiente, se ponen en relevancia las cualidades que albergan los lenguajes estéticos como alternativas para dar respuesta a las necesidades de reconfiguración de los procesos educativos, atendiendo a los retos y desafíos de la sociedad actual.

Desde este punto de vista, las propuestas metodológicas de los procesos artísticos permiten una aproximación al currículo a partir de múltiples perspectivas, al tener la capacidad de renovar los contenidos y dar cabida a ramificaciones del conocimiento en función de las inquietudes individuales y colectivas, lo que desemboca en diversas maneras de investigar, crear, experimentar y readecuar los saberes según lo que precise la acción educativa.

Por otro lado, uno de los atributos de los distintos lenguajes estéticos radica en la capacidad de generar diversos niveles discursivos, que dan lugar a una transformación en la manera en la que se comparten y reciben los contenidos, amplificando la experiencia pedagógica, que se ve enriquecida en sus dimensiones estéticas y semióticas. Esta cualidad retórica posibilita que se reduzca la brecha existente entre las distintas áreas del conocimiento y la cultura popular o las nuevas tecnologías de la comunicación.

Como consecuencia, se percibe la necesidad de que el colectivo docente reciba procesos de formación que se relacionen con las artes, no solo para ampliar sus destrezas técnicas relacionadas con la percepción y la creación a partir de los múltiples lenguajes, necesarias para mediar y proponer iniciativas transformadoras, sino para que se redimensionen sus propias capacidades cognitivas, emocionales, sociales, lúdicas, reflexivas, críticas y todas aquellas que subyacen en la esencia de la experiencia estética.

En definitiva, se trata de poner en valor las virtudes de la educación artística para suscitar actitudes y puntos 
de vista que originen diversos modos de abordar los conocimientos, múltiples formas de construir los discursos y de compartir los aprendizajes, diferentes maneras de acercamiento al acto creador, distintos procesos para construir y expresar los saberes; es decir, permitir que la heterogeneidad de los diversos lenguajes estéticos de pie a una pedagogía sensible y transformadora.

\section{Referencias}

Abad, J. (2021). Un compromiso con la contemporaneidad del arte y la educación. Arteterapia. Papeles de arteterapia y educación artística para la inclusión social, 16, 225-226. https://dx.doi. org/10.5209/arte.72950

Bildi, \& Escola Eina (2020). Bauhaus Distributed School and Bauhaus Pop-up School. https://www. bildi.net/projects/idees/215

Blocona, L. (2014). El Vorkurs de la Bauhaus. El Boomeran (g). Pastiche, (9). http://www.elboomeran.com/upload/ficheros/noticias/03.04_ el_vorkurs_de_la_bahaus.pdf

Bordes, J. (2006). La infancia del artista o las fuentes del Nilo. Real Academia de Bellas Artes de San Fernando.

Caeiro-Rodríguez, M. (2018). Aprendizaje Basado en la Creación y Educación Artística: proyectos de aula entre la metacognición y la metaemoción. Arte, Individuo y Sociedad, 30(1), 159-177. https://doi.org/10.5209/ARIS.57043

Caram, C. (2015). El currículum por proyecto. En $F$. d.-U. Palermo, Reflexión Académica en Diseño y Comunicación NoXXVI (pp. 92-94). Universidad de Palermo.

Caro, S. (2014). Valores Estéticos en el Diseño. Universidad de Palermo.

Diez del Corral, P. (2005). Una nueva mirada a la educación artística desde el paradigma del desarrollo humano. Universidad Complutense de Madrid.

Eco, U. (2007). Historia de la fealdad. Lumen.

Efland, A., Freedman, K. \& Stuhr, P. (2003). La educación en el arte posmoderno. Paidós.
Farina, C. (2006). Arte, cuerpo y subjetividad: experiencia estética y pedagogía. Educación Física y Ciencia, 8, 1-14. https://www.efyc.fahce.unlp. edu.ar/article/download/EFyCv08a04/5660/

Gómez, S. N., Gallo, L. E. \& Planella, J. (2018). Una educación poética del cuerpo o de lenguajes estético pedagógicos. Arte, Individuo y Sociedad, 30(1), 179-194. https://doi.org/10.5209/ARIS. 57351

González-Méndez, A. (2017). Capítulo 1. El área de educación artística en Primaria. Experimentación. En M. Caeiro-Rodríguez, Descubrir el arte: Formación en artes plásticas y visuales para maestros de Primaria. UNIR editorial.

Hoyuelos, A. (2013). Cada uno crece sólo si es sońado. En V. Vecchi, Arte y creatividad en Reggio Emilia. El papel de los talleres y sus posibilidades en la educación infantil (pp. 11-31). Ediciones Morata, S. L.

Marín, R. (2009). Didáctica de la eduación artística. Pearson.

Martínez-Agut, P. \& Ramos, C. (2015). Escuelas Reggio Emilia y los 100 lenguajes del nińo: experiencia en la formación de educadores infantiles. En U. d.-U. Catalunya, Actas del XVIII Coloquio de Historia de la Educación. Vol. 2. Sección 3 (pp. 139-151). Universitat de Vic-Universitat Central de Catalunya.

Martínez Vérez, M. V., Abad Molina, J., Mesias Lema, J. M. (2017). Arquitecturas del pensamiento: el lugar de las palabras. Arteterapia. Papeles de arteterapia educación para inclusión social, 12, 109-123. https://doi.org/10.5209/ ARTE. 57565

Mediavilla, E. (2021). Construcciones del cuerpo y las artes para una educación infantil transformadora. Arteterapia. Papeles de arteterapia y educación artística para la inclusión social, 16, 23-32. https://doi.org/10.5209/arte.71778

Mesías, J. M. (2018). Artivismo y compromiso social: Transformar la formación del profesorado desde la sensibilidad. Comunicar: Revista Cientifica Iberoamericana de Comunicación y Educación, 26(57), 19-28. https://doi.org/10.3916/ C57-2018-02 
Morin, E. (1999). Los siete saberes para una educación del futuro. Organización de las Naciones Unidas para la Educación, la Ciencia y la Cultura.

Planella, J. (2017). Pedagogía de lo sensible: subjetividades encarnadas en la escuela. Revista Reflexão e Ação, 25(3), 418-437. http://dx.doi. org/10.17058/rea.v25i3.8401

Pozo, M. \& Mayoral, E. (2017). Coincidencias pedagógicas social. Arquitectura y Espacio Social, (30), 41-62, http://hdl.handle.net/2117/117052

Rodari, G. (2001) Gramática de la fantasia. Introducción al arte de inventar historias. Editorial Planeta, S. A.

Ros, N. (2004). El lenguaje artístico, la educación y la creación. Revista Iberoamericana de Educación, 35(1), 1-8. https://doi.org/10.35362/rie 3512901
Sánchez-Carreño, J. \& Pérez-Rodríguez, C. (2011). Hacia un currículo transdisciplinario: una mirada desde el pensamiento complejo. Revista de Teoría y Didáctica de las Ciencias Sociales, (17), 143-164. https://www.redalyc.org/pdf/ 652/ 65221619010.pdf

Sánchez-Fernández, S., Pérez de Guzmán, V., Rebolledo-Gámez, T. \& Rodríguez-Casado, R. (2019). La cultura de paz y conflictos: implicaciones socioeducativas. Collectivus, Revista de Ciencias Sociales, 6(1), 235-250. https:// doi.org/10.15648/Coll.1.2019.13

Segni Mossi. (12 de agosto de 2021). About us. https://www.segnimossi.net/en/about

Vecchi, V. (2013). Arte y creatividad en Reggio Emilia. El papel de los talleres en la educación infantil y sus posibilidades. Ediciones Morata. 
